\title{
Answers to letter regarding our paper, "Medial branch neurotomy in low back pain" by Dr. S. Masala et al.
}

\author{
Salvatore Masala • Giovanni Nano • \\ Matteo Mammucari • Stefano Marcia • \\ Giovanni Simonetti
}

Received: 12 December 2011 / Accepted: 13 December 2011 / Published online: 24 December 2011

(C) Springer-Verlag 2011

Dear Sir,

We have read the comments about our work, and we are inclined to believe that all the matter has arisen from the term "pulsed" referred to radiofrequency, which appears twice in the Abstract and Introduction, that is a misnomer. We did not employ pulses of radiofrequency alternating current but single 70-s lasting shots of continuous radiofrequency alternating current for each lesion. As clearly described in the Technique section, we never mentioned any "pulse" there, what we produced were six contiguous lesions by heating tissues at $80^{\circ} \mathrm{C}$ for $70 \mathrm{~s}$ thus accomplishing an $8-9 \mathrm{~mm}$ wide $20 \mathrm{~mm}$ long whole lesion by moving the electrode tip in the described fashion. All the considerations about physics, histologic effect and clinical effectiveness of pulsed radiofrequency have been rightly expressed by you. This solves also the other related drawn criticisms.

\footnotetext{
S. Masala $\cdot$ G. Nano $(\bowtie) \cdot$ M. Mammucari $\cdot$ G. Simonetti

Department of Diagnostic and Molecular Imaging, Interventional

Radiology and Radiotherapy University "Tor Vergata",

81, Oxford Street,

00133 Rome, Italy

e-mail: gionano@gmail.com

S. Marcia

Department of Diagnostic and Molecular Imaging,

S. Giovanni di Dio Hospital,

Cagliari, Italy
}

Concerning the follow-up, we examined the patients at 1 week, but according to our general experience, we opted not to trust in their reports on pain relief at so close time interval because of the possible interference of unpredictable confounding factors. However matter stands, pain relief was immediate without a significant peak effect.

Our intention was not to study the cost ineffectiveness of the controlled diagnostic blocks, that has been well established in literature as you mention, but simply to obtain such highly selected patient population on which test the effectiveness of our technique. Alcoholization has been mentioned only for knowledge of a possible alternative treatment modality, proven ineffective, without deep examination.

Conflict of interest We declare that we have no conflict of interest. 